\title{
Eruption of a Twisted Filament and Associated Phenomena
}

\author{
Wahab Uddin and V. K. Verma \\ Uttar Pradesh State Observatory, NainiTal-263129, India
}

\begin{abstract}
In this paper we present CCD observations between February 14-20, 1994 and analysis of the giant twisted filament evolved in the active region NOAA 7671 . The dynamic eruption of the filament was accompanied by a major flare (3B/M4), CME, long duration type II, IV radio bursts, great microwave bursts, a long duration soft X-ray burst, SIDs, strong geomagnetic storms and a very energetic proton flare. We analysed and estimated the twist, length, volume, mass and energy associated with filament system between February 14 and 20, 1994. The present study shows that the magnetic energy required for the solar flare came from the filament system associated with the solar flare and associated phenomena.
\end{abstract}

\section{Introduction}

The study of filament/prominence evolution and its subsequent eruption has great significance in the field of solar activity for understanding the process of magnetic energy storage and its later release. The morphology of the filament reflects the complexity of the magnetic field and the energy storage site in the active region. When an active region is born, there might be transient process of energy libration associated with the emergence of magnetic flux. After the initial relaxation is over, the flare productive active region continue to grow and develop a highly distorted or sheared magnetic field cofiguration. This energy buildup phase can be regarded as a slow evolution of magnetic equilibria. The capacity of energy storage depends on the stability of the magnetic field, i.e., the magnetic field must be stable for smaller perturbations so that a large amount of energy can be stored. This phase is followed by an explosive liberation of the stored magnetic energy when the magnetic field loses equilibrium. In this phase the phenomenon of sudden filament disappearance is a familiar one to observe in $\mathrm{H} \alpha$. The disappearance of $\mathrm{H} \alpha$ dark filaments occurring over magnetic inversion lines is often associated with X-ray brightenings and sometimes with coronal mass ejections (CMEs). The disappearance may be caused by heating or by the dynamical processes similar in many respects to flares. The disparition brusque (DB) of an active filament often precedes the likely occurence of flares. It is widely assumed that flares draw their energy from the free energy of the magnetic field in the volume of the flare, the free energy being stored. For the occurrence of the large flares about $10^{32} \mathrm{ergs}$ of energy is required. Such a large amount of energy is stored in an active region in which active region filaments play an important role in the occurence of violent flares. Keeping in mind these facts, the study of the morphological evolution of active region filaments is very 
useful for understanding the energy storage process and its release during flares.

The present paper deals with observations of the evolution of a giant circular and twisted filament and its subsequent eruption on February 20, 1994 leading to a large solar flare and transient phenomena.

\section{Observations, Analysis and Conclusions}

The observations of the filament evolution in $\mathrm{H} \alpha$ emission between February 14 and 20, 1994 have been recorded with a $15 \mathrm{~cm} \mathrm{f} / 15$ Coude' refractor and Peltier-cooled $\left(-25^{\circ} \mathrm{C}\right) \mathrm{CCD}$ camera system. The CCD camera system used for the observations has a chip size of $385 \times 578$ pixels, with a pixel size $22 \times 22$ microns and a 16 bit A-D system.

The shape of the filament kept on changing from February 14-19, 1994 and, ultimately, the filament's twisting, shearing and energy buildup reached a critical stage which led to eruption of the filament system on February 20, 1994. The eruption of the filament was not recorded at UPSO because it took place before sunrise at NainiTal, India. The filament eruption and flare were recorded at Hida Observatory, Japan (Kurakawa and Shinkawa 1995). We recorded the decay phase of the filament/flare eruption.

In Figure 1 we show a composite figure of the filament evolution with the spot group from February 14-20, 1994. As is clear from Figure 1, the twisting and shearing of the filament continuously increased from February 14-20. The twisting of the filament started on February 14 and continued up to February 20 before the filament eruption. The filament erupted out on February 20 and the decay phase of the filament/flare eruption is shown in Figure 1. From the observations of filament evolution from February 14-19, we estimated the twist, length, mass, volume and energy of the filament which are shown in Table 1. The spot group associated with the filament under study between February 1420 was found to be rotating, which enhanced the twist in the filament. The emerging flux regions also increased the complexity of the magnetic field. The rotation of the sunspot group measured by us is shown in Table 1.

Table 1. Estimated parameters of the filament

\begin{tabular}{|c|c|c|c|c|c|c|c|}
\hline $\begin{array}{c}\text { Date } \\
\text { Feb. } 1994\end{array}$ & $\begin{array}{l}\text { Twist of } \\
\text { Filament } \\
\text { (in } \pi \text { ) }\end{array}$ & $\begin{array}{l}\text { Rotation } \\
\text { of Sunspots } \\
\text { in degree }\end{array}$ & $\begin{array}{l}\text { Length of } \\
\text { Filament } \\
\times 10^{5} \mathrm{~km}\end{array}$ & $\begin{array}{l}\text { Radius of } \\
\text { Filament } \\
\times 10^{3} \mathrm{~km}\end{array}$ & $\begin{array}{l}\text { Energy of } \\
\text { Filament } \\
\times 10^{32} \text { ergs }\end{array}$ & $\begin{array}{l}\text { Volume of } \\
\text { Filament } \\
\times 10^{28} \mathrm{~cm}^{3}\end{array}$ & $\begin{array}{l}\text { Mass of } \\
\text { Filament } \\
\times 10^{16} \mathrm{gm}\end{array}$ \\
\hline 14 & - & $\overline{0}$ & 1.677 & $4 . \overline{246}$ & 0.945 & $0 . \overline{95}$ & 1.63 \\
\hline 15 & 2 & 5 & 2.924 & 5.366 & 2.636 & 2.65 & 2.52 \\
\hline 16 & 2.5 & 15 & 3.268 & 6.449 & 4.247 & 4.27 & 3.46 \\
\hline 17 & 3.0 & 22 & 3.354 & 6.990 & 5.120 & 5.15 & 4.43 \\
\hline 18 & 3.5 & 32 & 3.440 & 7.512 & 6.070 & 6.10 & 5.14 \\
\hline 19 & 4.0 & 65 & 3.569 & 7.519 & 6.309 & 6.34 & 7.15 \\
\hline 20 & - & - & 3.660 & 7.524 & 6.48 & 6.51 & 8.57 \\
\hline
\end{tabular}

In the table the values of various parameters on February 20, 1994 are extrapolated values. The magnetic energy storage took place due to enhancement of twisting and shearing in the filament (Moore 1988 and Hagyard et al. 1990). This is a very dynamic, long duration event which occurred as the result of a 
twisted giant filament erupting. This was the one of the most energetic events in the decay phase of solar cycle 22 , because the event was associated with a major flare, 3B/M4, the third largest proton flare $(10,000 \mathrm{pfu})$ of solar cycle 21 and 22 , great wicrowave bursts, long duration type II $(1400 \mathrm{~km} / \mathrm{s})$ and IV radio bursts, SIDs, strong geomagnetic storms, a CME and other related phenomena (SGD 1994 and 1996). This is a good example of a dynamic disparition brusque. It is also the observational manifestation of the flare models proposed by Carmichel (1964), Sturrock (1968), Hirayama (1974) and Kopp and Pneuman (1976) (CSHKP model).

Kurokawa and Shinkawa (1995) reported that the mass ejected after the filament eruption had a velocity $>500 \mathrm{~km} / \mathrm{sec}$. We estimated the magnetic energy associated with filament system and the kinetic energy released after filament eruption using the following formulae:

$$
\begin{aligned}
& \frac{B^{2}}{8 \pi} V=6.5 \times 10^{32} \mathrm{erg} \\
& \frac{1}{2} M v^{2}=1.1 \times 10^{32} \mathrm{erg}
\end{aligned}
$$

assuming $B=500$ Gauss, $V=$ estimated volume of the filament, $M=$ mass of the filament and $\mathrm{v}=500 \mathrm{~km} / \mathrm{sec}$.

From the above it is clear that the magnetic energy associated with the filament system was greater than the kinetic energy released during the filament eruption. Therefore, we are of the view that the source of energy required for the flare was stored in the filament system associated with the flare.

Acknowledgments. We are thankful to the Organizers of IAU Colloquium 167 and the Indian National Science Academy, New Delhi, India for providing financial assistance for travel and local suppport.

\section{References}

Carmichael, H. 1964 in AAS-NASA Symposium on the Physics of Solar Flares, W. N. Hess (ed.), NASA SP-50, p. 451

Hagyard, M.J., Venkatakrishnan, P., and Smith, J.B. Jr. 1990, ApJS, 73, 159

Hirayama, T. 1974, Solar Phys., 34, 324

Kopp, R.A. and Pneuman, G.W. 1976, Solar Phys., 50, 85

Kurokawa, H. and Shinkawa, M. 1995, STEP GBRSC NEWS, 5/1, p. 3

Moore, R.L. 1988, ApJ, 324, 1132

Solar Geophysical Data 1994/1996, WDC-A, Boulder

Sturrock P.A. 1968, in Structure and Development of Solar Active Regions, K.O. Kiepenheuer (ed.), D. Reidel, Dordrecht, Holland, p. 471 


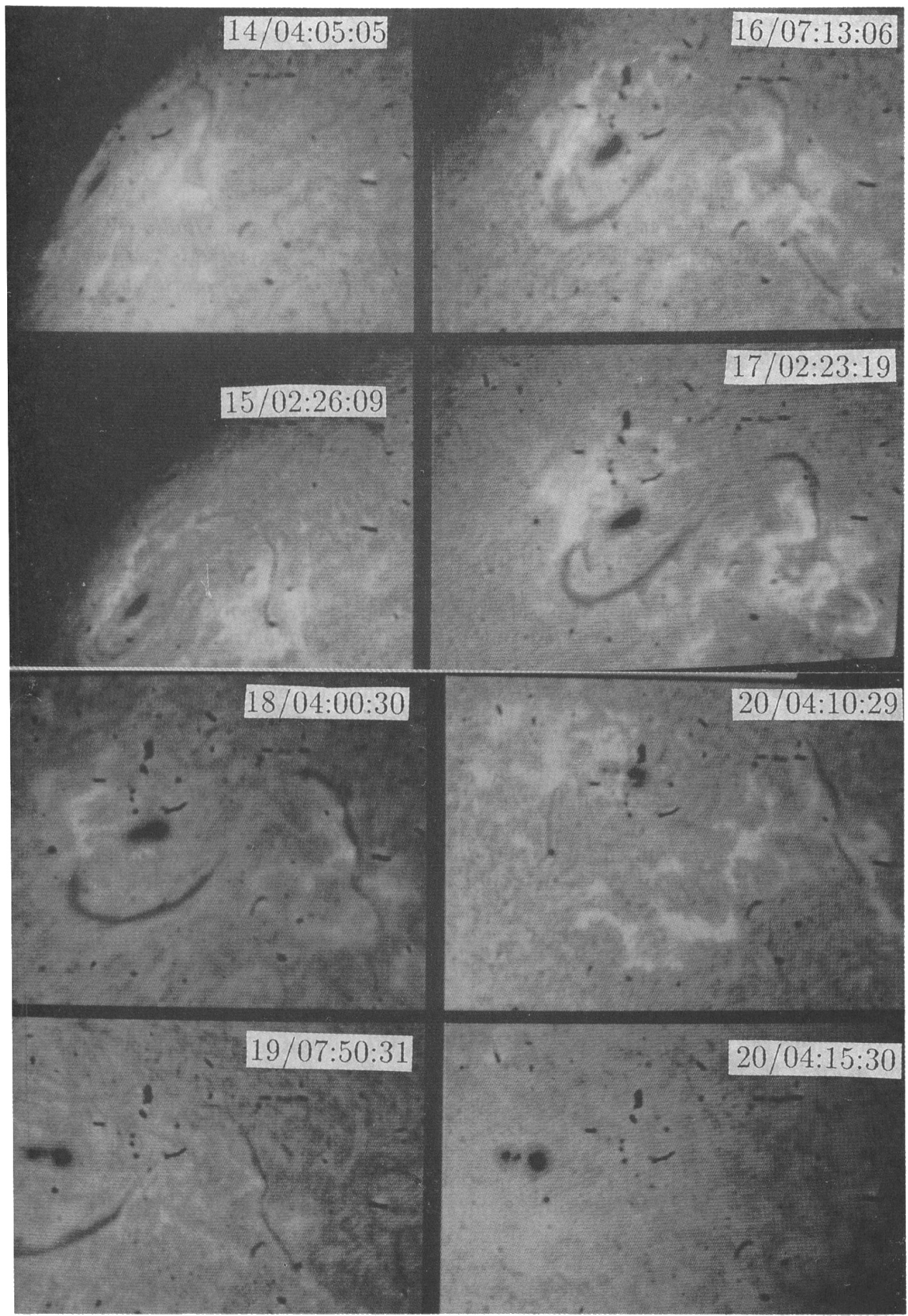

Figure 1. Filament evolution from February 14-20, 1994. 